https://doi.org/10.15407/dopovidi2020.07.017

UDC 512.544

\author{
V.A. Chupordia ${ }^{1}$, L.A. Kurdachenko ${ }^{2}$, N.N. Semko ${ }^{3}$ \\ 1,2 Oles Honchar Dnipro National University \\ ${ }^{3}$ University of State Fiscal Service of Ukraine, Irpin \\ E-mail: vchupordia@gmail.com, lkurdachenko@i.ua,dr.mykola.semko@gmail.com
}

\title{
On the structure of Leibniz algebras, whose subalgebras are ideals or core-free
}

Presented by Academician of the NAS of Ukraine A.M. Samoilenko

An algebra $L$ over a field $F$ is said to be a Leibniz algebra (more precisely, a left Leibniz algebra), if it satisfies the Leibniz identity: $[[a, b], c]=[a,[b, c]]-[b,[a, c]]$ for all $a, b, c \in$ L. Leibniz algebras are generalizations of Lie algebras. A subalgebra $S$ of a Leibniz algebra $L$ is called core-free, if $S$ does not include the non-zero ideal. We study the Leibniz algebras, whose subalgebras are either ideals or core-free.

Keywords: Leibniz algebra, Lie algebra, ideal, core-free subalgebras, monolithic algebra, extraspecial algebra.

Let $L$ be an algebra over a field $F$ with the binary operations + and [,]. Then $L$ is called a Leibniz algebra (more precisely, a left Leibniz algebra), if it satisfies the Leibniz identity

$[a,[b, c]]=[[a, b], c]+[b,[a, c]]$ for all $a, b, c \in L$.

Note that any Lie algebra is obviously a Leibniz algebra. Conversely, if $L$ is a Leibniz algebra such that $[a, a]=0$ for every element $a \in L$, then $L$ is a Lie algebra. Therefore, Lie algebras can be characterized as the Leibniz algebras, in which $[a, a]=0$ for every element $a$.

Leibniz algebras appeared first in papers by A.M. Blokh [1-3], in which he called them the $D$-algebras. However, in that time, those works were not in demand, and they had not been properly developed. Only after two decades, a real interest in Leibniz algebras arose. It was happened due to the work by J.L. Loday [4] (see also [5, Section 10.6]), who "rediscovered" these algebras and used the term Leibniz algebras, since it was Leibniz who discovered and proved the Leibniz rule for the differentiation of functions.

A Leibniz algebra, which is not a Lie algebra, has one specific ideal. By Leib $(L)$, we denote the subspace generated by the elements $[a, a], a \in L$. It is possible to prove that $\operatorname{Leib}(L)$ is an ideal of $L$. Moreover, $L / \operatorname{Leib}(L)$ is a Lie algebra. Conversely, if $H$ is an ideal of $L$ such that $L / H$ is a Lie algebra, then $\operatorname{Leib}(L) \leqslant H$.

Ци ту вання: Chupordia V.A., Kurdachenko L.A., Semko N.N. On the structure of Leibniz algebras, whose subalgebras are ideals or core-free. Допов. Наи. акад. наук Укр. 2020. № 7. С. 17-21. https://doi.org/10.15407/ dopovidi2020.07.017 
The ideal Leib $(L)$ is called the Leibniz kernel of an algebra $L$.

One approach to the study of Leibniz algebras, which proved to be quite effective, especially for infinite-dimensional Leibniz algebras, is to consider Leibniz algebras, all whose subalgebras have some fixed natural properties. This approach has been very effective for Lie algebras, while, in Leibniz algebras, it was used only recently. Thus, the Leibniz algebras, whose subalgebras are Lie algebras and Leibniz algebras with Abelian subalgebras, were studied in [6]. The Leibniz algebras, whose subalgebras are ideals, were considered in [7, 8].

Two ideals are naturally associated with each subalgebra $A$ of a Leibniz algebra $L$ : the ideal $A^{\mathrm{L}}$, which is the intersection of all ideals including $A$ (i.e., an ideal generated by $A$ ); and the ideal $\operatorname{Core}_{L}(A)$, which is the sum of all ideals that are contained in $A$.

A subalgebra $A$ of $L$ is called a core-free in $L \operatorname{Core}_{L}(A)=\langle 0\rangle$. From the definition, it follows that the core-free subalgebras are natural antipodes to the concepts of ideals. Therefore, the study of Leibniz algebras, whose subalgebras are either core-free or ideals, seems to us very natural. The main results of the current article give a description of such Leibniz algebras.

The first example of such algebras are Leibniz algebras, whose subalgebras are ideals. If every subalgebra of a Leibniz algebra $L$ is an ideal, then either $L$ is Abelian or $L=Z \oplus E$, where $Z \leqslant \zeta(L)$, and $E$ is an extraspecial subalgebra such that $[a, a] \neq 0$ for each element $a \in E \backslash \zeta(L)$ [7].

In particular, $[L, L] \leqslant \zeta(L)$, and a derived subalgebra $[L, L]$ has dimension 1 .

Recall that a Leibniz algebra $E$ is called extraspecial, if $\zeta(E)=[E, E]$ is a subalgebra of dimension 1.

Note also that a Lie algebra, whose subalgebras are ideals, is Abelian.

On the other hand, if $L$ is a simple Leibniz algebra, then its every proper subalgebra is corefree. We note that, in this case, $L$ is a Lie algebra.

We show also another example, which is typical in some sense. Let $L$ be a cyclic nilpotent Leibniz algebra of dimension 3. That is, $L=F a \oplus F b \oplus F c$, where $b=[a, a], c=[a, b]$. Here, $\operatorname{Leib}(L)=F b \oplus F c$. If $A$ is a subalgebra of $L$ such that $\operatorname{Leib}(L)$ does not include $A$, then $A=L$. If $A \leqslant \operatorname{Leib}(L)$ and $F c \leqslant A$, then $A$ is an ideal of $L$. If $A$ does not include $F c$, then $A$ is not an ideal, $\operatorname{dim}_{F}(A)=1$; therefore, $\operatorname{Core}_{L}(A)=\langle 0\rangle$.

Let $L$ be a Leibniz algebra. The intersection of all non-zero ideals $\operatorname{Mon}(L)$ of $L$ is called the monolith of a Leibniz algebra $L$. If $\operatorname{Mon}(L) \neq\langle 0\rangle$, then the Leibniz algebra $L$ is called monolithic, and, in this case, $\operatorname{Mon}(G)$ is the least non-zero ideal of $L$.

The description of Leibniz algebras, whose subalgebras are ideals or core-free, leads to the following natural cases:

$L$ is a non-monolithic Leibniz algebra;

$L$ is a monolithic Leibniz algebra.

The second case is basic, as shows

Theorem A. Let L be a non-monolithic Leibniz algebra. If every subalgebra of L, which is not an ideal, is core-free, then every subalgebra of is an ideal.

Corollary. Let L be a non-monolithic Lie algebra. If every subalgebra of $L$, which is not an ideal, is core-free, then $L$ is Abelian.

The monolithic case splits naturally in two subcases:

Leibniz algebra $L$ has a non-zero center;

Leibniz algebra $L$ has a zero center. 
Recall that the center $\zeta(L)$ of $L$ is defined by the rule:

$\zeta(L)=\{x \in L \mid[x, y]=0=[y, x]$ for each element $y \in L\}$.

The center is an ideal of $L$. In particular, we can consider the factor-algebra $L / \zeta(L)$.

More precisely, the left (respectively, right) center $\zeta^{\text {left }}(L)$ (respectively, $\zeta^{\text {right }}(L)$ ) of a Leibniz algebra $L$ is defined by the rule:

$$
\zeta^{\text {left }}(L)=\{x \in L \mid[x, y]=0 \text { for each element } y \in L\}
$$

(respectively,

$$
\left.\zeta^{\text {right }}(L)=\{x \in L \mid[y, x]=0 \text { for each element } y \in L\}\right) .
$$

It is not hard to prove that the left center of $L$ is an ideal, but this is not true for the right center. Moreover, $\operatorname{Leib}(L) \leqslant \zeta^{\text {left }}(L)$, so that $L / \zeta^{\text {left }}(L)$ is a Lie algebra. The right center is an subalgebra of $L$, and, in general, the left and right centers are different; they even may have different dimensions. Paper [9] contains some examples, which show this.

Theorem B. Let L be a Leibniz algebra. Suppose L includes a subalgebra, which is not an ideal, and every subalgebra of $L$, which is not an ideal, is core-free. If the center of $L$ is non-zero, then $L$ satisfies the following conditions:

(i) $L$ is monolithic and $\operatorname{Mon}(L)=\zeta(L)=\gamma_{3}(L)$, in particular, $\operatorname{dim}_{F}(\zeta(L))=1$;

(ii) $\gamma_{2}(L)=[L, L] \leqslant \zeta_{2}(L)$ and $\gamma_{2}(L)$ has dimension 2 ;

(iii) every subalgebra of $L$, which is not an ideal, is Abelian;

(iv) every subalgebra of $L / \zeta(L)$ is an ideal.

Conversely, if $L$ is a Leibniz algebra satisfying the above conditions, then every subalgebra of $L$ either is core-free or an ideal.

Corollary. Let L be a monolithic non-Abelian Lie algebra having a non-trivial center. Then every subalgebra of $L$, which is not an ideal, is core-free, if and only if $L$ is an extraspecial algebra.

The situation, where $L$ has a non-central monolith, is considered in a following

Theorem C. Let L be a monolithic Leibniz algebra, whose center is zero. Suppose that every subalgebra of $L$, which is not an ideal, is core-free. If $L$ is not a Lie algebra, then the following conditions hold;

(i) $\operatorname{Mon}(L)$ is a minimal ideal of $L$;

(ii) $\operatorname{Mon}(L)$ is a maximal Abelian ideal of $L$;

(iii) $L=\operatorname{Mon}(L) \oplus A$ for some Abelian subalgebra $A$;

(iv) $\operatorname{Ann}_{L}(\operatorname{Mon}(L))=\operatorname{Ann}_{L}{ }^{\text {left }}(\operatorname{Mon}(L))=\operatorname{Mon}(L)$.

Conversely, if $L$ is a Leibniz algebra satisfying the above conditions, then every subalgebra of $L$ either is core-free or an ideal.

We note also that, in this case, a core-free subalgebra can be not Abelian. The following example shows this.

Example. Let $F$ be an arbitrary field, and let $L$ be a vector space over $F$ with a basis $\{a, b$, $\left.a_{1}, a_{2}\right\}$. Define the operation [,] on $L$ in the following way:

$$
[a, a]=a_{1},\left[a, a_{1}\right]=a_{2},\left[a, a_{2}\right]=-a_{1}-a_{2},[a, b]=0,
$$




$$
\begin{aligned}
& {[b, a]=a_{1}+a_{2},[b, b]=0,\left[b, a_{1}\right]=-a_{1},\left[b, a_{2}\right]=-a_{2},} \\
& {\left[a_{1}, a\right]=0,\left[a_{1}, b\right]=0,\left[a_{2}, a\right]=0,\left[a_{2}, b\right]=0,} \\
& {\left[a_{1}, a_{1}\right]=0,\left[a_{1}, a_{2}\right]=0,\left[a_{2}, a_{1}\right]=0,\left[a_{2}, a_{2}\right]=0 .}
\end{aligned}
$$

It is possible to check that $L$ is a Leibniz algebra, $\operatorname{Leib}(L)=F a_{1}+F a_{2}$, Leib $(L)=\operatorname{Mon}(L)$, a factor-algebra $L / \operatorname{Mon}(L)$ is Abelian. Proposition 1 implies that every subalgebra of $L$, which is not an ideal, is core-free. But the subalgebra $\left\langle b, a_{1}\right\rangle$ is not an ideal, is not Abelian and is core-free.

For Lie algebras, we obtained the following

Proposition. Let L be a monolithic Lie algebra, whose center is zero. Suppose that every subalgebra of $L$, which is not an ideal, is core-free. Then $\operatorname{Mon}(L)$ is a minimal ideal of $L$ such that $\operatorname{Ann}_{L}(\operatorname{Mon}(L))=\operatorname{Mon}(L)$, and the factor-algebra L/Mon $(L)$ is Abelian. Moreover, every core-free subalgebra of $L$ is Abelian.

If $\operatorname{Mon}(L)$ is Abelian, the description is more detailed.

Let $L$ be a Leibniz algebra, and let $a$ be a some fixed element of $L$. Consider the mapping $\boldsymbol{r}_{a}$ : $L \longrightarrow L$ defined by the rule $\boldsymbol{r}_{a}(x)=[x, a], x \in L$. It is not hard to see that $\boldsymbol{r}_{a}$ is a linear mapping, $\beta \boldsymbol{r}_{a}=\boldsymbol{r}_{\beta a}$ and $\boldsymbol{r}_{a}+\boldsymbol{r}_{b}=\boldsymbol{r}_{a+b}$ for all $a, b \in L$ and $\beta \in F$. Put $\boldsymbol{c}_{a}(x)=x+[x, a], x \in L$, i.e., $\boldsymbol{c}_{a}(x)=$ $=i+\boldsymbol{r}_{a}(x)$, where $i$ is an identity permutation of $L$. Clearly, $\boldsymbol{c}_{a}$ is also a linear mapping.

Theorem D. Let L be a monolithic Lie algebra, whose center is zero. Suppose that every subalgebra of $L$, which is not an ideal, is core-free. If the monolith of $L$ is Abelian, then the following conditions hold;

(i) $\operatorname{Mon}(L)$ is a minimal ideal of $L$;

(ii) $\operatorname{Mon}(L)$ is a maximal Abelian ideal of $L$;

(iii) $\operatorname{Ann}_{L}(\operatorname{Mon}(L))=\operatorname{Mon}(L)$;

(iv) $L=\operatorname{Mon}(L) \oplus$ A for some Abelian subalgebra $A$;

(v) if $L=\operatorname{Mon}(L) \oplus C$ for some subalgebra $C$, then there exists an element $v \in \operatorname{Mon}(L)$ such that $C=\boldsymbol{c}_{v}(A)$. Moreover, $\boldsymbol{c}_{v}$ is an automorphism of algebra $L$.

\section{REFERENCES}

1. Blokh, A. (1965). A generalization of the concept of a Lie algebra. Dokl. AN SSSR, 165, No. 3, pp. 471-473 (in Russian).

2. Blokh, A. (1967). Cartan-Eilenberg homology theory for a generalized class of Lie algebras. Dokl. AN SSSR, 175, No. 2, pp. 266-268 (in Russian).

3. Blokh, A. M. (1971). A certain generalization of the concept of Lie algebra. Uchenye zapiski Moskov. Gos. Pedagog. Inst., 375, pp. 9-20 (in Russian).

4. Loday, J. L. (1993). Une version non commutative des algebres de Lie: les algèbres de Leibniz. Enseign. Math., 39, pp. 269-293. https://doi.org/10.5169/seals-60428

5. Loday, J. L. (1998). Cyclic homology. Grundlehren der Mathematischen Wissenschaften, Vol. 301. 2nd ed. Berlin: Springer. https://doi.org/10.1007/978-3-662-11389-9

6. Chupordya, V. A., Kurdachenko, L. A. \& Subbotin, I. Ya. (2017). On some "minimal" Leibniz algebras. J. Algebra Appl., 16, 1750082. https://doi.org/10.1142/S0219498817500827

7. Kurdachenko, L. A., Semko, N. N. \& Subbotin, I. Ya. (2017). The Leibniz algebras whose subalgebras are ideals. Open Math., 15, pp. 92-100. https://doi.org/10.1515/math-2017-0010

8. Kurdachenko, L. A., Semko, N. N. \& Subbotin, I. Ya. (2017). Leibniz algebras, whose all subalgebras are ideals. Dopov. Nac. akad. nauk. Ukr., No. 6, pp. 9-13. https://doi.org/10.15407/dopovidi2017.06.009 
9. Kurdachenko, L. A., Otal, J. \& Pypka, A. A. (2016). Relationships between factors of canonical central series of Leibniz algebras. European J. Math., 2, pp. 565-577. https://doi.org/10.1007/s40879-016-0093-5

Received 09.01.2020

\section{В.А. Чупордя ${ }^{1}$, Л.А. Курдаченко ${ }^{2}$, М.М. Семко ${ }^{3}$}

1,2 Дніпровський національний університет ім. Олеся Гончара

${ }^{3}$ Університет державної фіскальної служби України, Ірпінь

E-mail: vchupordia@gmail.com, lkurdachenko@i.ua, dr.mykola.semko@gmail.com

\section{ПРО СТРУКТУРУ АЛГЕБР ЛЕЙБНІЦА, УСІ ПІДАЛГЕБРИ} ЯКИХ Є ІДЕАЛАМИ АБО ВІЛЬНИМИ ВІД ЯДРА

Алгебра $L$ над полем $F$ називається алгеброю Лейбніца (точніше, лівою алгеброю Лейбніча), якщо вона задовольняє таку тотожність Лейбніца: $[[a, b], c]=[a,[b, c]]-[b,[a, c]]$ для всіх $a, b, c \in L$. Алгебри Лейбніца являють собою узагальнення алгебр Лі. Підалгебра $S$ алгебри Лейбніца $L$ називається вільною від ядра, якщо $S$ не містить ненульових ідеалів. Розглянуто алгебри Лейбніца, усі підалгебри яких є ідеалами або вільними від ядра.

Ключові слова: алгебра Лейбніча, алгебра Лі, ідеал, підалгебра, вільна від ядра, монолітична алгебра, екстраспеціальна алгебра. 